\title{
ESTIMATION OF THE THICKNESS AND EMULSION RATE OF OIL SPILLED AT SEA USING HYPERSPECTRAL REMOTE SENSING IMAGERY IN THE SWIR DOMAIN
}

\author{
G. Sicot ${ }^{\mathrm{a} *}$, M. Lennon ${ }^{\mathrm{a}}$, V. Miegebielle ${ }^{\mathrm{b}}$, D. Dubucq ${ }^{\mathrm{b}}$ \\ ${ }^{a}$ SAS Actimar - 29200 Brest, France - (sicot, lennon)@actimar.fr \\ b TOTAL E\&P - 64000 Pau - (veronique.miegebielle, dominique.dubucq)@ total.com
}

KEY WORDS: hyperspectral, oil spill, thickness, emulsion rate

\begin{abstract}
:
The thickness and the emulsion rate of an oil spill are two key parameters allowing to design a tailored response to an oil discharge. If estimated on per pixel basis at a high spatial resolution, the estimation of the oil thickness allows the volume of pollutant to be estimated, and that volume is needed in order to evaluate the magnitude of the pollution, and to determine the most adapted recovering means to use. The estimation of the spatial distribution of the thicknesses also allows the guidance of the recovering means at sea. The emulsion rate can guide the strategy to adopt in order to deal with an offshore oil spill: efficiency of dispersants is for example not identical on a pure oil or on an emulsion. Moreover, the thickness and emulsion rate allow the amount of the oil that has been discharged to be estimated. It appears that the shape of the reflectance spectrum of oil in the SWIR range (1000-2500nm) varies according to the emulsion rate and to the layer thickness. That shape still varies when the oil layer reaches a few millimetres, which is not the case in the visible range (400-700nm), where the spectral variation saturates around $200 \mu \mathrm{m}$ (the upper limit of the Bonn agreement oil appearance code). In that context, hyperspectral imagery in the SWIR range shows a high potential to describe and characterize oil spills. Previous methods which intend to estimate those two parameters are based on the use of a spectral library. In that paper, we will present a method based on the inversion of a simple radiative transfer model in the oil layer. We will show that the proposed method is robust against another parameter that affects the reflectance spectrum: the size of water droplets in the emulsion. The method shows relevant results using measurements made in laboratory, equivalent to the ones obtained using methods based on the use of a spectral library. The method has the advantage to release the need of a spectral library, and to provide maps of thickness and emulsion rate values per pixel. The maps obtained are not composed of regions of thickness ranges, such as the ones obtained using discretized levels of measurements in the spectral library, or maps made from visual observations following the Bonn agreement oil appearance code.
\end{abstract}

\section{INTRODUCTION}

Oil spill detection at sea using remote sensing techniques is now used for monitoring of illegal discharges and accidents (Leifer et al., 2012). However the characterization of the spills is still a field of research. Nowadays oil spills are usually characterized through a visual segmentation by using the Bonn agreement oil apparence code (Agreement Bonn Counter Pollution Manual, 1999). Hyperspectral remote sensing has shown some interesting results in the VNIR range (400 to $1000 \mathrm{~nm}$ ) for a rough estimation of thickness and the evaluation of volume at sea using airborne sensors (Lennon et al., 2006, Otremba and Piskozub, 2004, Wettle et al., 2009). During the Deepwater Horizon oil spill, Clark et al. (2010) have shown in (Clark et al., 2010) the high potential of hyperspectral data in the SWIR range $(1000 \mathrm{~nm}$ to $2500 \mathrm{~nm})$ for the estimation of high thickness ranges (over a millimetre) and emulsion rates. They proceed by associating shape parameters of reflectance spectra of the data to those from a spectral library. Here, we will present a method that prevents from the use of a reflectance spectral library. That method also allows maps of thickness and emulsion rate values per pixel to be obtained. Those maps are then not composed of regions of thickness or emulsion rate ranges.

This study is part of a research and development project that aims to assess the ability of the hyperspectral imagery to detect, characterize, and identify the pollutants in an oil spill. The characterization here consists in estimating the thickness of the pollutant layer and the emulsion rate of the mixture oil/water that compose the oil spill.

\footnotetext{
${ }^{*}$ Corresponding author
}

We will first recall the tools we have in hand: a spectral library and a model that simulate the reflectance spectra of a thin layer of an oil/water emulsion in the SWIR spectral range (Sicot et al., 2014). The laboratory measurements were performed with the parameters of the acquisition all under control. All those laboratory measurements are collected in the spectral library that will be briefly presented in section 2 . The model is presented in section 3 . It shows that the size of water droplets in the emulsion is a key parameter that modifies the shape of the reflectance spectra. Unfortunately that model appears complicated to invert and we will propose in section 4 . another formulation to be used for the estimation of the thickness and of the emulsion rate of the oil layer. The method is applied on data obtained from airborne data acquisition campaigns and from the spectral library. Airborne data acquisition campaigns were carried out over real controlled oil spills at sea. Those oil discharges were organized off the coasts of Norway by the Norwegian Clean Seas Association for Operating Companies (NOFO). The last section is devoted to the analysis of the results on those data as well as on data from the Deepwater Horizon oil spill.

\section{A SPECTRAL LIBRARY}

The goal when building the spectral libray was to reproduce the observations presented in (Clark et al., 2010) and to extend the measurements on other products. The library includes the transmittance and reflectance spectra of thin layers of seven mineral 
oils ${ }^{1}$, two dispersants ${ }^{2}$, two vegetal oils ${ }^{3}$ and two dissolved algaes $^{4}$.

These measurements were realized in collaboration with the Cedre (French Centre of Documentation, Research and Experimentation on Accidental Water Pollution). Some weathering process simulations were done using a pollution weathering tank (the socalled 'polludrome') on a crude oil (PL222U). The emulsion type was controlled thanks to the use of different processes to produce those emulsions: some are produced using the polludrome (slow agitation), some using rotative flasks (slow to medium agitation), and some others using an ultra-turrax ${ }^{\circledR}$ (quick agitation). The reflectance spectra are measured on the different emulsion types. The variation of the shape of the reflectance spectra with respect to the type of emulsion in the SWIR spectral range is put into evidence thanks to those measurements. Those observations lead us to the development of a simple radiative transfer model that intends to simulate the reflectance spectra. That model will be briefly described in the next section.

\section{A RADIATIVE TRANSFER MODEL IN THE OIL LAYER}

The development of the model is inspired by the models developed in the water color domain (Mobley, 2010, Mobley, 1994, Morel, 1973). It is described by the illustration shown on the figure 1 .

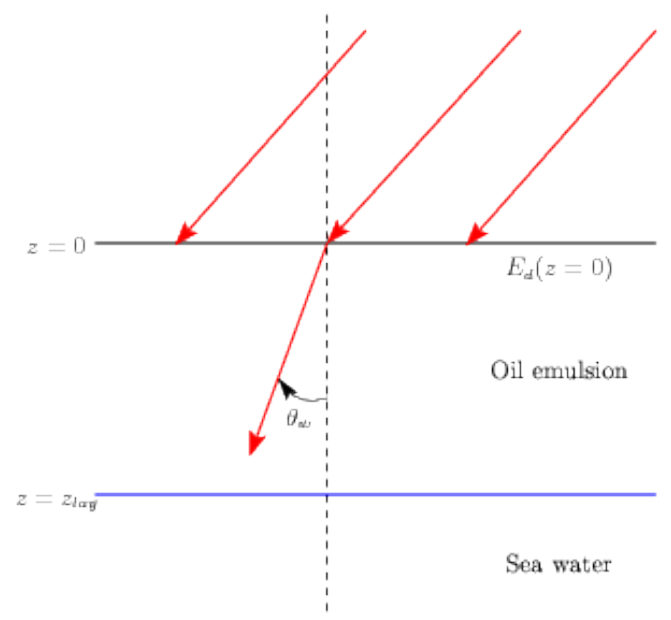

Figure 1: Illustration of the model developped in (Sicot et al., 2014) and the notations used

As shown on that figure, the model consists in a flat emulsion layer above a flat sea water surface. In the model, we consider that the sea water is clear, has an infinite depth and that the oil/ water emulsion layer is homogeneous. The model takes the assumption that the main reflective phenomena is due to the scattering of the light by water droplets in the emulsion layer. A resolution of the radiative transfer equation can be found in (Sicot et al., 2014). Based on some approximation (the Quasi Single Scattering Approximation), a semi-analytic solution can be written as follows:

\footnotetext{
${ }^{1}$ Three crude oils from West Africa, TOTAL Fluid Gazoil, SPRES Gazoil, SPRES bunker fioul, and the oil used by NOFO during the controlled discharge at sea

${ }^{2}$ OSR 52 and OSR 62

${ }^{3}$ palm oil and sunflower oil

${ }^{4} \mathrm{~A}$ brown and a green algae
}

$$
\begin{array}{r}
r_{r s}(\theta, \phi)=r_{r s}^{\infty}(\theta, \phi)\left[1-e^{\left(\frac{1}{\mu}-\frac{1}{\mu_{w}}\right) c z_{l a y}}+\right. \\
A_{1}(\theta, \phi)\left(e^{-\frac{2 c z_{l a y}}{\mu_{w}}}-e^{\left(\frac{1}{\mu}-\frac{1}{\mu_{w}}\right) c z_{l a y}}\right)+ \\
\left.A_{2}(\theta, \phi)\left(e^{\left(\frac{1}{\mu}-\frac{1}{\mu_{w}}\right) c z_{l a y}}-e^{\frac{2 c z_{l a y}}{\mu}}\right)\right]
\end{array}
$$

with

$$
\begin{gathered}
r_{r s}^{\infty}(\theta, \phi)=\omega_{0} \frac{\tilde{\beta}\left(\left(\mu_{w}, \phi_{w}\right) \rightarrow(\mu, \phi)\right)}{\mu_{w}-\mu} \\
A_{1}=\rho\left(\theta_{w}\right)\left(\frac{\mu_{w}-\mu}{\mu_{w}+\mu}\right)\left(\frac{\tilde{\beta}\left(\left(-\mu_{w}, \phi_{w}\right) \rightarrow(\mu, \phi)\right)}{\tilde{\beta}\left(\left(\mu_{w}, \phi_{w}\right) \rightarrow(\mu, \phi)\right)}\right) \\
A_{2}=\rho(\theta)\left(\frac{\mu_{w}-\mu}{\mu_{w}+\mu}\right)\left(\frac{\tilde{\beta}\left(\left(\mu_{w}, \phi_{w}\right) \rightarrow(-\mu, \phi)\right)}{\tilde{\beta}\left(\left(\mu_{w}, \phi_{w}\right) \rightarrow(\mu, \phi)\right)}\right)
\end{gathered}
$$

where

$$
\begin{aligned}
& r_{r s} \text { is the remote sensing reflectance } \\
& \theta, \phi \text { are the angles of acquisition } \\
& c \text { is the diffuse attenuation coefficient } \\
& \mu=\cos \theta \text { and } \mu_{w}=\cos \theta_{w} \\
& z_{l a y} \text { is the thickness of the layer } \\
& \omega_{0} \text { is the single scattering albedo } \\
& \tilde{\beta} \text { is the normalised volume scattering function } \\
& \rho \text { is the reflexion coefficient of the interface } \\
& \text { oil/sea water }
\end{aligned}
$$

In order to lighten the notations, we do not explicitly show the dependance to wavelengths. In (Sicot et al., 2014), the parameters used to describe the scattering phenomena, the scattering part of $c$ and $\tilde{\beta}$, are calculated according to the Lorenz-Mie theory.

The figure 2 shows normalized reflectance spectra calculated from the model using an emulsion rate of $60 \%$, while the figure 3 shows reflectance spectra presented in (Clark et al., 2010). The model shows some great similarities with laboratory measurements. The model is able to reproduce the behavior of the reflectance spectra according to the thickness and to the emulsion rate (Sicot et al., 2014), and thus to show the "wide variety of shape" of the reflectance spectra, as stated in (Clark et al., 2010). The analysis of the reflectance spectra produced by the model also shows that the size of the water droplets in the emulsion is a key parameter in the reflectance spectra shape. In order to model the size of the water droplets in the emulsion layer, a function that represents the distribution of the water droplets size is introduced. In the figure 2, a lognormal distribution has been used for this purpose.

\section{THE PROPOSED METHOD FOR THE ESTIMATION OF THE PARAMETERS}

In this section, we will propose a method to estimate the thickness and the emulsion rate from a reflectance spectra. At first, we will recall the objectives and the context of its application.

\subsection{Objectives and context of use}

The proposed method is designed to describe an oil spill by estimating its thickness and its emulsion rate. In this study, the data used is hyperspectral imagery in the SWIR range. The estimation is made on a per pixel basis, the expected results will then be 


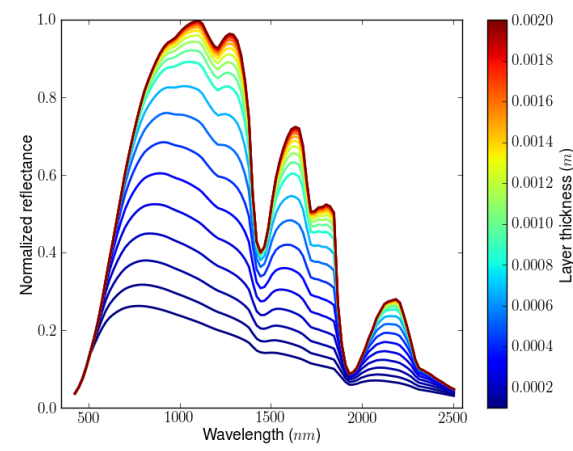

Figure 2: Normalized reflectance spectra calculated from the model using an emulsion rate of $60 \%$

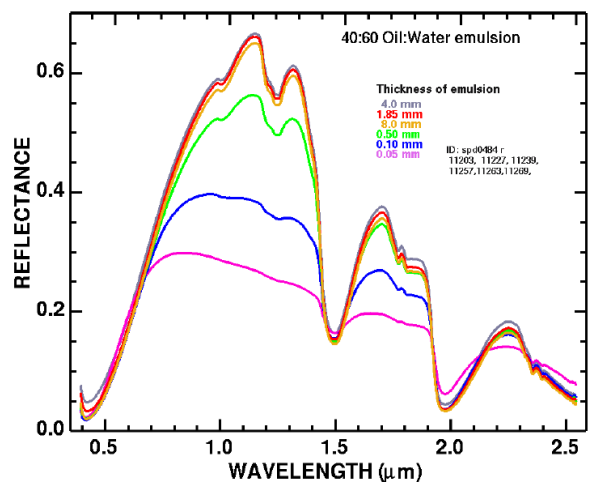

Figure 3: Example of reflectance spectra presented in (Clark et al., 2010)

maps of thicknesses and emulsion rates over the extent of the oil spills.

Hyperspectral is a passive remote sensing mean and uses the sun light as energy source. The characteristics of the atmosphere in the SWIR range should be taken into account. The atmospheric transmittance is presented on figure 4. That figure shows that the SWIR range has two opaque spectral bands and some others that are quite non-transparent due to presence of water and carbon dioxide in the atmosphere. The wavelengths around 1100nm, from $1350 \mathrm{~nm}$ to $1500 \mathrm{~nm}$ and from $1800 \mathrm{~nm}$ to $1950 \mathrm{~nm}$ are not usable. The proposed approach should take care of this characteristics to be operationnally applied.

\subsection{The proposed method}

Our first intention was to use the model proposed in (Sicot et al., 2014) which can be summarized by the expression (1). It appears that the inversion of that model is relatively complicated due to the need to estimate the distribution of water droplets size from the reflectance spectra. The computation load would be too heavy and that makes this idea inappropriate in an operational context. We then propose to use a simplest model which would still be relevant to estimate the thickness and the emulsion rate of the oil spill, and which would in the same time be relatively invariant to the distribution of the water droplets size.

The model proposed for this purpose is described here below:

$$
\begin{array}{r}
r_{r s}(\lambda)=A \exp \left(-\gamma\left(\tau a_{\text {water }}(\lambda)+(1-\tau) a_{\text {oil }}(\lambda)\right) z_{\text {lay }}\right) \\
\text { for } \lambda_{\min } \leq \lambda \leq \lambda_{\max }
\end{array}
$$



Figure 4: Atmospheric transmittance in the VNIR and the SWIR

\begin{tabular}{|c|c|}
\hline where & $\begin{array}{l}\lambda \text { is the wavelength } \\
A, \gamma \text { are constants } \\
\tau \text { is the emulsion rate }\end{array}$ \\
\hline & $\begin{array}{l}z_{\text {lay }} \text { is the thickness of the emulsion layer } \\
a_{\text {water }}(\lambda) \text { is the absorption of the water } \\
a_{\text {oil }}(\lambda) \text { is the absorption of the oil }\end{array}$ \\
\hline
\end{tabular}
range (Richter and Schlapfer, 2012)

The expression in (5) is inspired by the Beer-Lambert law. Thereafter, we will consider that the absorption of the water, $a_{\text {water }}(\lambda)$, and of the oil, $a_{o i l}(\lambda)$, are known. The parameter $\gamma$ is a constant factor also considered as known. $\lambda_{\min }$ and $\lambda_{\max }$ will be choosen in order to be appropriate considering the atmosphere absorption. The other parameters $A, \tau, z_{l a y}$ are the ones that have to be estimated by the method.

The estimation is performed thanks to an optimization method. The results presented in the next section are computed by using the Levenberg-Marquardt algorithm ${ }^{5}$. Oil shows 3 main absorptions bands in the SWIR (Clark et al., 2009): one at 1200nm, another one at $1730 \mathrm{~nm}$ and the last one at $2300 \mathrm{~nm}$. The spectral range around the absorption at $1730 \mathrm{~nm}$ is chosen for the estimation. That spectral range appears to be the best compromise between the standard signal to noise ratio of sensors in the SWIR range and the effect of the emulsion rate and thickness variation on the spectral shape. Due to the atmosphere attenuation, we set $\lambda_{\min }$ to $1500 \mathrm{~nm}$ and $\lambda_{\max }$ to $1750 \mathrm{~nm}$.

In the next section, we will evaluate the relevance of the expression given in (5) to estimate the oil spill thickness and the emulsion rate.

\section{RESULTS}

This section is dedicated to the assessment of the relevance of the expression given in (5) for the characterization of an oil spill. This task will be divided into three parts. The first part will be devoted to the analysis of the robustness of the estimator with respect to the statistical distribution of the water droplets size. This will be performed by using the model presented in section 3 . The second part will use the measurements made in laboratory. Finally in the third part we will apply our method on a real dataset acquired during the Deepwater Horizon oil spill and on dataset acquired during the controlled discharges organized by the NOFO.

\footnotetext{
${ }^{5}$ we use the implementation included in the fortran library minpack
} 


\subsection{Robustness with respect to the water droplets size}

The protocol used for building the spectral library does not include any quantitative measurement of the size of the water droplets in the emulsion, and does not include spectra enough to be used for analyzing the robustness of the estimator with respect to the water droplets size. In order to assess that robustness, we will use the model briefly presented in the section 3 . In that model, the statistical distribution of the water droplets has to be parameterized. Since we did not find any previous works analyzing the distribution of water droplets in oil, we looked at models used for the parameterization of the distribution of air droplets or particulates in water (Zhang et al., 2011). The two main statistical distributions used are the Junge distribution, which is similar to the power law distribution, and the lognormal distribution. In our simulations, we found that reflectance spectra computed using the lognormal distribution were more similar to the measurements made in laboratory than those computed with a Junge law. The lognormal distribution is defined by two parameters: $\mu$ and $\sigma$. The expression of the lognormal distribution is given here below:

$$
f(x)=\frac{1}{x \sigma \sqrt{2 \pi}} \exp \left(-\frac{(\ln x-\mu)^{2}}{2 \sigma^{2}}\right)
$$

The two parameters are the mean and the standard deviation of the logarithm of the random variable defined by the lognormal distribution. In this study, we will focus our analysis on the robustness of our estimator with respect to the parameter $\mu$.

The results of the estimation of the thickness of the emulsion layer is presented on figure 5 , while the results of the estimation of the emulsion rate is presented on figure 6 . The results show that the estimation of the emulsion rate seems to be independant of the size of water droplets. The figure 6 shows that the proposed method however underestimates a little the emulsion rate. We can also note that the method fails to estimate the emulsion rate when the layer thickness is too thin.

The analysis concerning the estimation of the thickness is less straigthforward. Please note that the parameter $\gamma$ has been set to 1 to get the results. The results show that the estimation of the thickness is correlated to the thickness computed by the model, regardless of the water droplets size. It also appears that the estimated thickness reaches a limit which depends on the emulsion rate and on the water droplets size. For example, on figure 5(a), a layer thicker than $5 \mathrm{~mm}$ is estimated, while on figure $5(\mathrm{c})$ the estimator reaches a limit around 200-300 $\mu \mathrm{m}$. It is interesting to recall that the upper limit of the Bonn agreement oil apparence code, which is nowadays operationnally used, is $200 \mu \mathrm{m}$. Over that limit, the thickness range can not be estimated using the Bonn agreement protocol.

\subsection{Results on the spectral library}

In this section, the proposed method is applied on the reflectance spectra from the spectral library. A thickness estimation limit is reached for most of the reflectance spectra, as observed in section 5.1. The results on the layer thickness thus do not appear relevant.

Results concerning the emulsion rate are presented on figure 7. The results are shown on two different oils: a crude oil on figure 7 (a) and the oil used during the controlled discharged organized by the NOFO on the figures 7(b) and (c). In order to evaluate the influence of water droplets size, we present results on emulsions produced in two ways: reflectance spectra presented on figure 7(b) were measured on emulsions produced using rotative flasks, while reflectance spectra presented on figure 7(c) were measured on emulsions produced using an ultra-turrax ${ }^{\circledR}$. On all those results, a very good correlation is found between the measured and estimated emulsion rates. The results also show that the emulsion rates produced using the ultra-turrax ${ }^{\circledR}$ are slightly underestimated (see figure $7(\mathrm{c})$ ), which is not the case on the emulsion rates produced using the rotative flasks (see figure 7(b)).

\subsection{Application on hyperspectral data}

The image presented in this section was acquired by the Jet Propulsion Laboratory (JPL) using an AVIRIS sensor. The data are available in radiance on the website of the aviris ${ }^{6}$. The image presented on figure 8(a) is part of the flight line called f100517t01p00 r11. This line was acquired on May, the $17^{\text {th }}, 2010$. This flight line has been chosen as it has already been processed by Clark $e t$ $a l$. and presented in (Clark et al., 2010). The first operation was to convert the radiance datacube into surface reflectance. The atmospheric correction has been realized using the ATCOR software which is based on MODTRAN. On this reflectance datacube we apply our method which gives us the results presented on figures 8(b) and (c). We use the results presented in (Clark et al., 2010) for a comparative analysis of our own results.

The estimation of the emulsion rate seems to be relevant, the estimation becoming more noisy however when the oil layer thickness becomes very thin. On the other hand, the estimation of the thickness layer presented on figure 8(b) overestimates compared to the results presented in (Clark et al., 2010). In order to reduce this overestimation, we can calibrate our method by setting $\gamma$ to 2. With this setting, the results ${ }^{7}$ match more with those of Clark et al. It has to be noticed that the estimation of the layer thickness and emulsion rate is made on a per pixel basis, which allows to get a continuous spatial evolution of the thickness and of the emulsion rate.

In order to show the robustness of the proposed method we have also applied the method on data acquired during the controlled discharges organized by the NOFO in june 2012. The VNIR spectral range being more adapted to characterize thin layers of oil, while the SWIR spectral range being more apppropriate on thick layers, a pre-processing has been performed in order to separate the oil spill into thin layers and thick layers parts. The proposed method has been applied only on the thick layers parts. The results are presented on figure 9 . The estimated thickness of the oil layer is often quite thin (below $1 \mathrm{~mm}$ ). The mean emulsion rate is estimated to be around $51 \%$ which is close to the real discharged oil emulsion rate of $60 \%$.

\section{CONCLUSION}

In this paper, we have presented a method which intends to estimate on a per pixel basis the thickness and the emulsion rate of an oil slick from hysperspectral imagery in the SWIR domain. That spectral range domain has been chosen due to the potential of those wavelengths to characterize oil spill shown by Clark $e t$ $a l$. Especially, it has been shown that thicknesses of oil layers up to several millimeters can be estimated. The method proposed by Clark et al. is strongly based on a spectral library. In this paper, we have presented the preliminary results of a method that intends to reduce the use of the spectral library by inverting a very simple model inspired by the Beer-Lambert law.

\footnotetext{
${ }^{6}$ http://aviris.jpl.nasa.gov/data/get_aviris_data. html

${ }^{7}$ not presented in this paper
} 


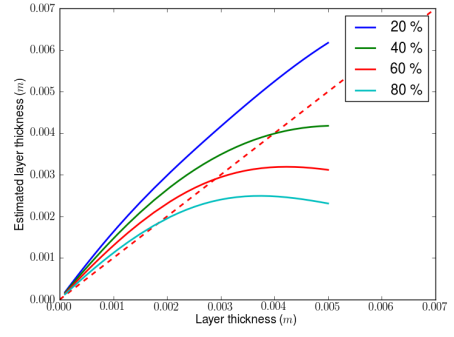

(a) Simulations performed with $\mu=5 \mu m$

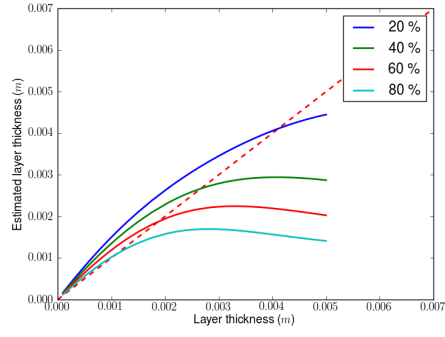

(b) Simulations performed with $\mu=1 \mu m$

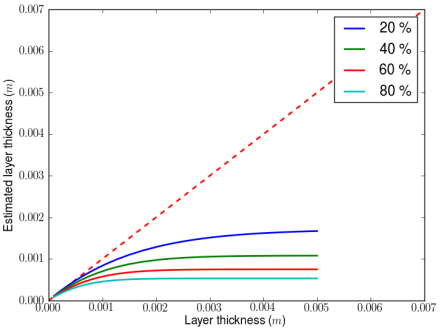

(c) Simulations performed with $\mu=0.2 \mu m$

Figure 5: Estimation of the thickness of the emulsion layer for several droplets size and various emulsion rates. For each figure, the distribution used for the model is the lognormal distribution with $\sigma=0.5$. The parameter $\mu$ used for the lognormal distribution is indicated in the caption of each figure. The dashed red line is the result of the ideal estimator.

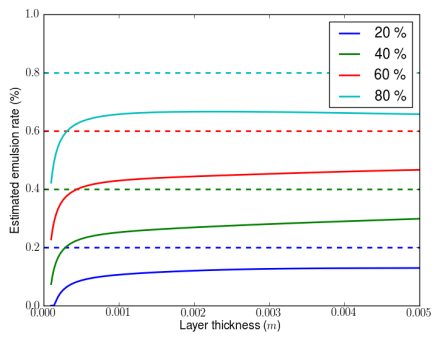

(a) Simulations performed with $\mu=5 \mu m$

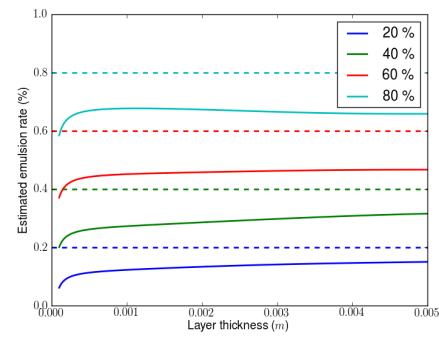

(b) Simulations performed with $\mu=1 \mu m$

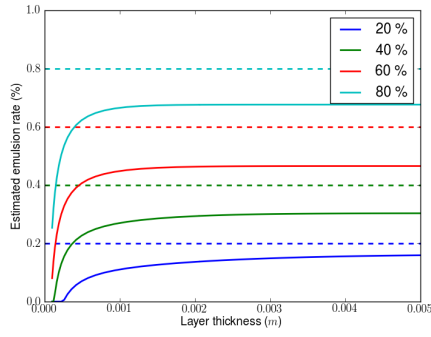

(c) Simulations performed with $\mu=0.2 \mu m$

Figure 6: Estimation of the emulsion rate of the emulsion layer for several droplets size and various emulsion rates. For each figure, the distribution used for the model is the lognormal distribution with $\sigma=0.5$. The parameter $\mu$ used for the lognormal distribution is indicated in the caption of each figure. The dashed lines are the results of the ideal estimator.

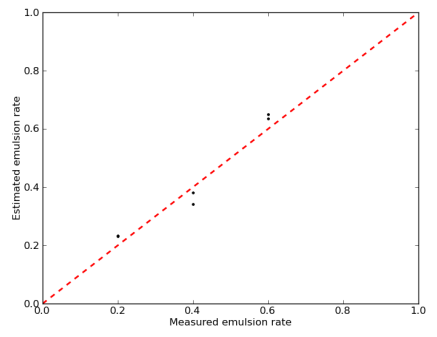

(a) Crude oil (B17G), emulsion performed with rotative flasks

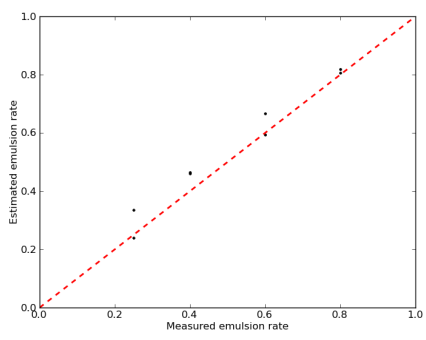

(b) oil used during the discharge organized by the NOFO, emulsion performed with rotative flasks

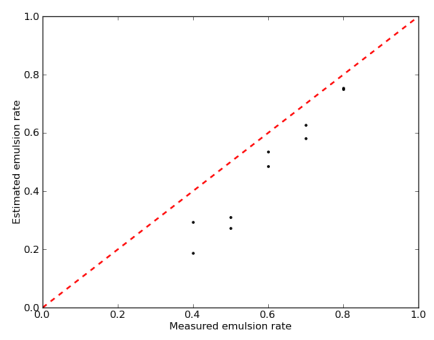

(c) oil used during the discharge organized by the NOFO, emulsion performed with ultraturrax ${ }^{\circledR}$

Figure 7: Estimation of the emulsion rate performed on two types of oil emulsion. Regarding the oil used during the controlled discharge organized by the NOFO, results are shown on emulsions produced in two ways, one using rotative flasks (figure (b)), the other one using an ultra-turrax ${ }^{\circledR}$ (figure (c)). The dashed lines are the results of the ideal estimator.

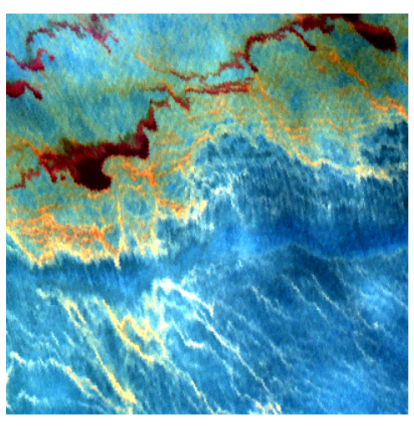

(a) Pseudo true color of hyperspectral data

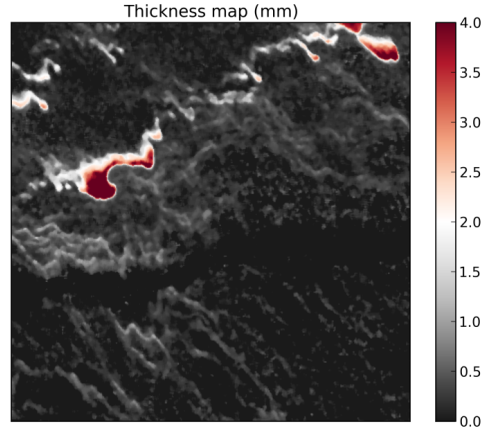

(b) Estimation of the oil thickness

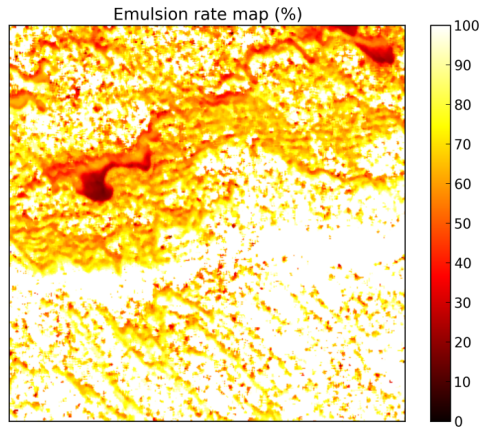

(c) Estimation of the emulsion rate

Figure 8: Application of our method on hyperspectral data acquired over the Deepwater Horizon oil spill by the JPL with an aviris sensor 


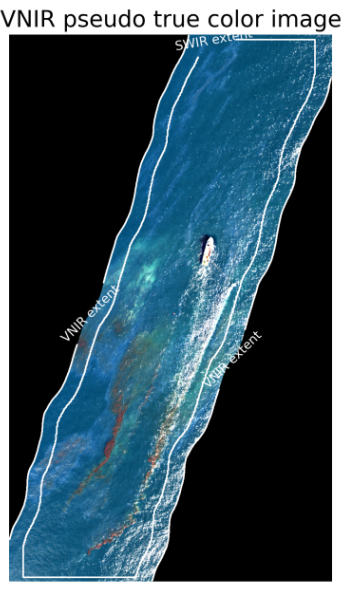

(a) Pseudo true color of hyperspectral data

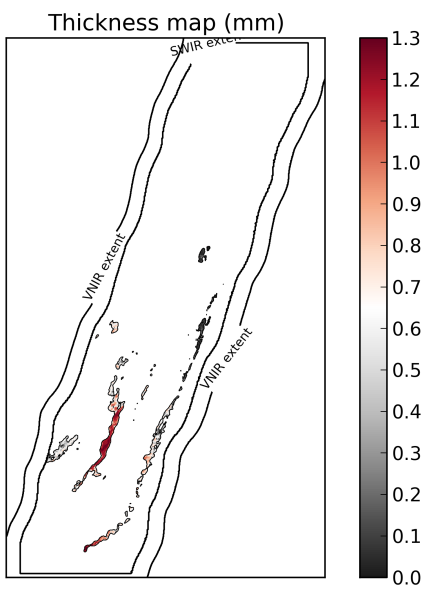

(b) Estimation of the oil thickness

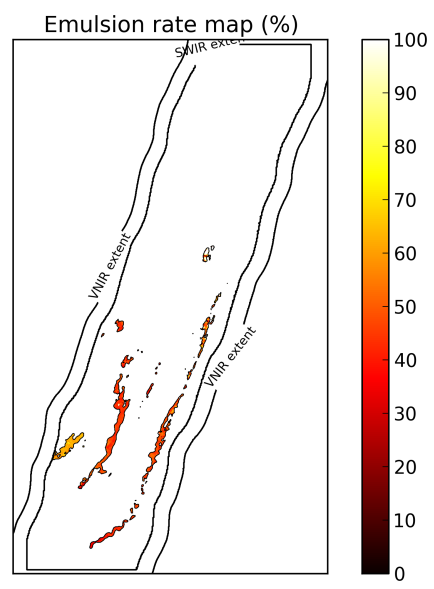

(c) Estimation of the emulsion rate

Figure 9: Application of our method on hyperspectral data acquired by Actimar during the controlled oil discharges organized by the NOFO

In order to assess the relevance of the simple model used we have proceeded in three steps. First we have evaluated the estimations of the two targeted parameters on reflectance spectra simulated from a more complete model developed before (Sicot et al., 2014). Although that model still needs improvements, it shows that the water droplets size in the emulsion is a key parameter in the reflectance spectral shape. In a second step we have evaluated the quality of the parameters estimated from our method thanks to a spectral library which includes several types of oil and different ways to produce emulsions. Finally we have tested our method on real datasets acquired over the Deepwater Horizon oil spill and over NOFO experiments at sea. The proposed method gives relevant results in much of the cases, and seems to be able to produce relatively reliable estimations of the thickness and of the emulsion rate of an oil slick. A calibration of the thickness estimate is potentially needed in some cases however, that has to investigated in further works.

The method proposed has the advantage to produce an estimation for each pixel of the image. As it is the result of an optimization process, the estimation is spatially continuous and does not depend on the content of a spectral library. This approach is innovative even in the depiction of the oil spill. Indeed, the present way to describe an oil spill generally consists in using the Bonn agreement oil appearence code, which is a manual segmentation of the oil spill according to the oil appearence which can be converted into layer thickness intervals. We hope this new way to describe oil spills will give more accurate information to evaluate the amount of pollutant discharged and the magnitude of a spill, as well as to improve the definition of the strategy to be used to face oil spills.

\section{ACKNOWLEDGEMENTS}

The authors would like to thank the Cedre for their support in the realization of the spectral library.

\section{REFERENCES}

Agreement Bonn Counter Pollution Manual, 1999.

Clark, R. N., Curchin, J. M., Hoefen, T. M. and Swayze, G. A., 2009. Reflectance spectroscopy of organic compounds: 1. alkanes. Journal of Geophysical Research: Planets (1991-2012).
Clark, R. N., Swayze, G. A., Leifer, I., Livo, K. E., Kokaly, R., Hoefen, T., Lundeen, S., Eastwood, M., Green, R. O., Pearson, N. et al., 2010. A method for quantitative mapping of thick oil spills using imaging spectroscopy. US Geological Survey Open-File Report 1167(2010), pp. 1-51.

Leifer, I., Lehr, W. J., Simecek-Beatty, D., Bradley, E., Clark, R., Dennison, P., Hu, Y., Matheson, S., Jones, C. E., Holt, B. et al., 2012. State of the art satellite and airborne marine oil spill remote sensing: Application to the bp deepwater horizon oil spill. Remote Sensing of Environment 124, pp. 185-209.

Lennon, M., Babichenko, S., Thomas, N., Mariette, V., Mercier, G. and Lisin, A., 2006. Detection and mapping of oil slicks in the sea by combined use of hyperspectral imagery and laser induced fluorescence. EARSeL eProceedings 5(1), pp. 120-128.

Mobley, C., 2010. Ocean optics web book.

Mobley, C. D., 1994. Light and water: Radiative transfer in natural waters. Vol. 592, Academic press San Diego.

Morel, A., 1973. Diffusion de la lumière par les eaux de mer. résultats expérimentaux et approche théorique. Optics of the Sea 61, pp. 3-1.

Otremba, Z. and Piskozub, J., 2004. Modelling the bidirectional reflectance distribution function (brdf) of seawater polluted by an oil film. Optics Express 12(8), pp. 1671-1676.

Richter, R. and Schlapfer, D., 2012. Atmospheric/topographic correction for airborne imagery: Atcor-4 user guide. DLR IB pp. 565-02.

Sicot, G., Lennon, M., Miegebielle, V. and Dubucq, D., 2014. Analysis of the reflectance spectra of oil emulsion spilled on the sea surface. In: SPIE Remote Sensing, International Society for Optics and Photonics, pp. 924007-924007.

Wettle, M., Daniel, P. J., Logan, G. A. and Thankappan, M., 2009. Assessing the effect of hydrocarbon oil type and thickness on a remote sensing signal: A sensitivity study based on the optical properties of two different oil types and the hymap and quickbird sensors. Remote Sensing of Environment 113(9), pp. 2000-2010.

Zhang, X., Twardowski, M. and Lewis, M., 2011. Retrieving composition and sizes of oceanic particle subpopulations from the volume scattering function. Applied optics 50(9), pp. 12401259. 